\title{
Editorial
}

\section{Propofol-related convulsions}

The possibility of delayed seizures after propofol anaesthesia could have a serious impact on its usefulness for ambulatory patients. Dr. Finley and his colleagues are the first anaesthetists in Canada to describe cases in which propofol has been implicated in the aetiology of convulsions. ' Their patients were two young teenagers who developed generalized tonic-clonic convulsions several hours after propofol-lidocaine-fentanyl mixtures were administered for bone marrow biopsy procedures. Although the authors acknowledge that a number of contributory factors could have led to these events, their concern that the use of propofol may provoke life-threatening complications after patients leave hospital cannot be ignored. Accordingly, evidence which supports or refutes the possibility of an association between propofol and convulsions has been examined.

Since its introduction into clinical practice in 1986, propofol has been given to over 15 million patients world wide and to 1.3 million patients in Canada in one year, 1989-90 (data provided by Zeneca, formerly ICI Pharma, and Statistics Canada). From 1987-92 there were 37 case reports of neurological excitatory events following propofol in the anaesthetic literature. These involved adults, of both sexes, aged 16-55 yr. Each exhibited some of a spectrum of neurological symptoms: grand-mal convulsions, opisthotonus, tonic-clonic movements, muscle rigidity, confusion, unconsciousness, athetoid movements and incontinence. These adverse neurological reactions were seen on induction of anaesthesia ${ }^{2,3}$ and at its termination before awakening, ${ }^{4}$ but predominantly within the first hour after the end of surgery. 5,6 Some were more remote from the procedure, occurring up to five days later. ${ }^{7}$ Most episodes were transient, lasting a few minutes and resolving spontaneously, although occasionally episodes persisted for $24 \mathrm{hr}$ and extended over as long as 23 days. When these case histories were reviewed, 11 patients were found to have histories of pre-existing epilepsy and 17 had received additional drugs known to have proconvulsant activity. Nevertheless, 13 cases remain in which no confounding factors could be found and, in

From the Departments of Anaesthesia, BC Children's Hospital and the University of British Columbia, Vancouver, B.C. the absence of any other explanation, focussed on propofol as the likely cause. The Committee on the Safety of Medicines in the United Kingdom estimated the incidence of seizures after propofol as 1 in 47,000 administrations from their reports, and issued an advisory warning of this possibility.

The potential for inducing convulsions iatrogenically with anaesthetic agents is not a new phenomenon. Clinical experiences before propofol was available associated ether with febrile convulsions; isoflurane with myoclonic seizures; ${ }^{8}$ barbiturates, especially methohexitone, with epileptogenic properties; promethazine, ${ }^{9}$ opioids ${ }^{10}$ and local anaesthetics ${ }^{11,12}$ with grand-mal seizures. Hypoxia was frequently cited as a cause, while sickle-cell disease and cerebral oedema due to fluid overload were suggested in isolated cases. More careful scrutiny of the effects of anaesthetic agents in the last $15 \mathrm{yr}$ has shown that some have predictable convulsant activity. Enflurane is recognized for increasing cortical activity and inducing convulsions in a dose-dependent biphasic pattern, ${ }^{13}$ suggesting that it has a mixture of depressant and convulsant properties. Generalized myotonic contractions have been reported during recovery from enflurane anaesthesia ${ }^{14}$ and convulsions may be delayed for 2-3 days after its administration. ${ }^{15}$ Fentanyl has been associated with tonic and clonic grand mal seizures in the absence of any changes in the electroencephalogram (EEG). ${ }^{16}$ Usually these have occurred during the administration of the drug, given in low or high doses, but may be during recovery. Interesting observations have been made on withdrawal seizures in mice exposed to nitrous oxide. 17,18 These seizures were maximal at 2-3 min of recovery and lasted for six hours. Opioid and endorphin mechanisms have been postulated and might explain why some patients have convulsions at different stages of recovery from other anaesthetic agents.

At first, propofol seemed to be a pure hypnotic with no convulsant or anti-convulsant actions. ${ }^{19}$ Later, it was shown in animal models to have anti-convulsant properties similar to thiopentone against electrically and drug induced seizures. ${ }^{20,21}$ This was borne out clinically as the duration of electroconvulsive therapy (ECT) seizures was shortened and the number of treatments required in- 
creased when propofol was administered. ${ }^{22,23}$ Finally, propofol was used successfully in the treatment of status epilepticus in patients who were refractory to established treatment regimens. ${ }^{24}$ Like thiopentone and diazepam, another anticonvulsant, propofol is believed to act by activating GABA receptors and enhancing transmission. ${ }^{25}$ In the spinal cord, propofol seems to be a glycine antagonist ${ }^{26}$ like strychnine, which could explain the unexpected spasms of opisthotonus and myoclonus described. Propofol also produces convulsions in mice in a biphasic dose-dependent manner, with seizures provoked by doses close to the anaesthetic dose, consistent with induction and emergence being the most likely times for convulsions to occur. Thus, in addition to its hypnotic role, a dual action of propofol in man is suggested: predominantly anticonvulsant with a weaker convulsant component.

Fascinating studies are emerging to demonstrate EEG changes in healthy patients receiving propofol. Brainstem auditory evoked responses are dose related with increased latencies as anaesthesia deepens. ${ }^{27}$ These changes are more like the volatile agents than other intravenous anaesthetics, but are too small to interfere with deafness testing when propofol is used for sedation. Seifert $e t$ al. found dose-related activation of beta-frequencies of the EEG occur with the onset of sedation and accompanying restlessness and agitation. ${ }^{28}$ These changes persist with deepening sedation even though the excitatory movements stop, but cease within $30 \mathrm{~min}$ of discontinuing the propofol infusion. Similar EEG features have also been seen with sedative doses of barbiturates and benzodiazepines, but not with opioids or etomidate. Jantti et al. identified a characteristic spindle on the burst suppression pattern of the EEG with propofol. ${ }^{29}$ This differs from the burst suppression described with enflurane, isoflurane and barbiturate anaesthesia and is benign and reversible, unlike the burst suppression seen in brain damaged patients. In children, spontaneous movements are a common feature on induction of anaesthesia with propofol. These are reduced from $100 \%$ to $14 \%$ by increasing the induction dose from 3 to $5 \mathrm{mg} \cdot \mathrm{kg}^{-1} i$. Borgeat et al. recorded the EEG during induction and saw activation with predominantly beta activity, without burst suppression, but with a transient period of delta waves coinciding with the movements. ${ }^{30}$ In the absence of EEG abnormalities they concluded that the spontaneous movements have a subcortical origin. When they followed the EEG in the recovery period in a six-week-old child, similar EEG patterns were seen for the $20 \mathrm{~min}$ period in which movements occurred, with a normal pattern $24 \mathrm{hr}$ later. ${ }^{31}$ They noted that the anti-emetic and anti-pruritic effects of low doses of propofol last much longer than hypnotic effects. If these are attributable to subcortical effects, then the phar- macodynamics of propofol may differ at that level from those at the cortical level, leading to the possibility of delayed and late phenomena that do not accord with the brief hypnotic effect and rapid elimination of propofol.

Propofol is the most recent anaesthetic drug to be implicated as a cause of seizures. It is unlikely that propofol gives rise to epileptiform convulsions, and the neurological manifestations described probably result from the same mechanisms that cause the excitatory effects on induction. If cortical epileptic foci are inhibited by subcortical activity, then it would be possible for propofol to release the epileptic seizure activity in susceptible patients. Unusual and concerning features are the athetoid movements and opisthotonus seen after anaesthesia. These are almost confined to propofol, although phenothiazines can give rise to dystonic reactions with opisthotonus and athetoid movements have been associated with midazolam sedation. As yet, it is difficult to relate the delayed convulsions reported directly to the use of propofol and any connection remains open to conjecture.

\section{Propofol et convulsions}

L'apparition de convulsions tardives après l'administration de propofol pourrait avoir des conséquences néfastes sur son utilisation en chirurgie ambulatoire. Au Canada, les observations du Drs Finley et de ses collègues sont les premières à mettre en cause le propofol dans le genèse de crises convulsives. ${ }^{1}$ Ils rapportent le cas de deux adolescents qui développent des crises convulsives généralisées tonicocloniques plusieurs heures après avoir reçu un mélange propofol-lidocaïne-fentanyl pour un prélèvement biopsique de la moelle osseuse. Les auteurs reconnaissent que plusieurs facteurs ont pu contribuer à cette complication mais ils ont raison de sinquiéter sur ces événements dangereux. Ils convient donc examiner l'évidence qui infirme ou confirme cette association propofol et convulsions.

Depuis le début de son introduction en clinique en 1986, le propofol a été administré à 15 millions de patients dans le monde et, au Canada pendant l'année 1989-90, à 1,3 million de patients (données fournies par Zeneca, autrefois ICI Pharma et statistiques Canada). De 1987 à 1992, la littérature rapporte 37 observations d'excitation neurogène consécutives à l'administration de propofol. Elles intéressent des adultes des deux sexes âgés de 16 à 55 ans. Ces patients ont tous montré des signes d'irritation neurologique: des convulsions de type grand mal, 
de l'opisthotonos, des mouvements tonicocloniques, de la rigidité musculaire, de la confusion, de l'inconscience, des mouvements athétoïdes et de l'incontinence. Ces réactions indésirables sont constatées à l'induction de l'anesthésie ${ }^{2,3}$ et à la fin, avant le réveil, ${ }^{4}$ mais surtout en deçà de la première heure qui suit l'anesthésie. ${ }^{5,6}$ Quelques unes surviennent jusqu'à cinq heures de l'intervention. ${ }^{7}$ La plupart des épisodes sont transitoires avec récupération spontanée, bien que certaines se prolongent pendant 24 heures et aussi longtemps que 23 jours. Après révision, on constate que 11 de ces patients ont des antécédents épileptiques et 17 ont reçu des drogues proconvulsivantes. Toutefois, dans treize cas, on ne trouve aucun facteur prédisposant, et à défaut d'autres explications, on pointe du doigt le propofol. Le comité de la sécurité des médicaments du Royaume-Uni estime que l'incidence des convulsions après propofol est d'une sur $\mathbf{4 7 0 0 0}$ administrations et a émis un avertissement à cet effet.

Le déclenchement iatrogène de convulsions ne se présente pas comme un phénomène nouveau en anesthésie. Avant l'arrivée du propofol, on associait l'éther à des convulsions fébriles; l'isoflurane à des attaques de myoclonie, ${ }^{8}$ le méthophexitone à des propriétés épileptogènes; la prométhazine, ${ }^{9}$ les opiacés ${ }^{10}$ et les anesthésiques locaux ${ }^{11,12}$ aux crises de type grand mal. On a souvent mentionné l'hypoxie comme agent causal, alors que l'anémie falciforme et l'oedème cérébral par surcharge liquidienne ont été suggérés dans des cas isolés. Au cours des 15 dernières années, un examen minitieux des effets des anesthésiques a montré que certains avaient une activité proconvulsivante. On sait que l'enflurane intensifie l'activité corticale et induit des crises biphasiques ${ }^{13}$ identiques à celles du grand mal et liées à la concentration, suggérant ainsi un mélange de propriétés proconvulsivantes et anticonvulsivantes. Avec l'enflurane ${ }^{14}$ des contractions myotoniques généralisées ont été rapportées pendant la phase de récupération et des convulsions peuvent être tardives, jusqu'à $2-3$ jours après l'administration. ${ }^{15}$ Le fentanyl est associé à des crises tonicocloniques de grand mal sans modifications électroencéphalographiques. ${ }^{16}$ Ordinairement elles surviennent pendant l'administration de la drogue, à faibles et fortes doses, mais peuvent aussi apparaître au réveil. Des observations de crises convulsives sont rapportées au sevrage du protoxyde d'azote chez des souris exposées à cet agent. ${ }^{17,18}$ Ces crises sont maximales 2-3 min avant le réveil et peuvent continuer pendant six heures. On a postulé que les opioïdes et les endorphines interviennent dans ce mécanisme, ce qui pourrait expliquer pourquoi certains patients présentent des convulsions à différents stages de la récupération avec d'autres agents anesthésiques.

On a cru au début que le propofol était un hypnotique pur sans activité proconvulsivante ni anticonvulsivante. ${ }^{19}$
Par la suite, sur des modèles animaux, on lui a trouvé des propriétés anticonvulsivantes semblables à celles du thiopentone sur les convulsions d'origine électrique et pharmacologique. ${ }^{20,21}$ Ceci s'est manifesté en clinique par une diminution de la durée des crises convulsives en sismothérapie et par l'augmentation du nombre de traitements requis lorsque le propofol est administré. ${ }^{21,23} \mathrm{De}$ plus on a utilisé du propofol avec succès pour le traitement du status epilepticus réfractaire au traitement habituel. ${ }^{24}$ Comme pour le thiopentone et le diazepam, lui-même anticonvulsivant, on croit que le propofol agit en activateur des récepteurs GABA facilitant ainsi la transmission. ${ }^{25} \mathrm{Au}$ niveau de la moelle épinière, le propofol semble antagoniser la glycine, ${ }^{26}$ comme la strychnine, ce qui peut expliquer l'opisthotonos et les myoclonies. De plus, le propofol induit chez la souris des convulsions biphasiques proportionnelles à la dose, voisines de la dose anesthésique, ce qui correspond avec l'apparition de convulsions à l'induction et au réveil. On prête ainsi une double activité au propofol: une prédominance anticonvulsivante avec une composante proconvulsivante faible.

Sous propofol, des études sur des patients non tarés montrent des modifications de l'EEG. Les potentiels évoqués auditifs du tronc cérébral diminuent avec l'augmentation de la dose et la latence croît avec l'approfondissement de l'anesthésie. ${ }^{27}$ Ces changements ressemblent plus à ceux des agents volatils que des agents intraveineux, mais sont trop légers pour parasiter les tests de surdité lorsque le propofol est utilisé pour la sédation. Seifert et al. ont trouvé qu'à l'EEG une activation des fréquences bêta survient au début de la sédation et s'accompagne d'instabilité psychomotrice et d'agitation. ${ }^{28} \mathrm{Ces}$ changements persistent lorsqu'on approfondit la sédation même après l'arrêt des mouvements, mais cessent dans les 30 minutes qui suivent l'arrêt de la perfusion de propofol. On retrouve des caractéristiques identiques à l'EEG avec des doses sédatives de barbituriques et de benzodiazépines, contrairement aux opiacés et à l'étomidate. Jantti et al. ont identifié un fuseau caractéristique sur le pattern de " burst suppression " de l'EEG avec le propofol. ${ }^{29}$ Ceci diffère du « burst suppresion " décrit avec l'enflurane, l'isoflurane et les barbituriques et est bénin et réversible, contrairement au "burst suppression " de la lésion cérébrale. Chez l'enfant, les mouvements spontanés sont une caractéristique habituelle de l'induction au propofol et ils diminuent de $100 \%$ à $14 \%$ lorsqu'on augmente la dose de 3 à $5 \mathrm{mg} \cdot \mathrm{kg}^{-1} i v$. Borgeat et al. ont enregistré l'EEG à l'induction et ont constaté l'activation d'une activité prédominante bêta, sans " burst suppression ", mais avec une période transitoire d'ondes delta qui coïncide avec les mouvements. ${ }^{30}$ En absence d'anomalies à l'EEG ils concluent à une origine sous corticale de mouvements spontanés. A la période postopéra- 
toire, sur l'EEG d'un bébé de six semaines, un tracé identique est observé par les mêmes auteurs lors de mouvements avec un retour à la normale 24 heures plus tard. ${ }^{31}$ Ils constatent que les effets anti-émétiques et antiprungeux de faibles doses de propofol durent plus longtemps que les effets hypnotiques. Si ces effets sont souscorticaux, la pharmacodynamique du propofol peut différer de ceux qui agissent au niveau cortical, ce qui pourrait faire penser à un phénomène retardé qui concorde mal avec le court effet hypnotique et l'élimination rapide du propofol.

Le propofol devient l'agent anesthésique le plus récent impliqué dans la génèse de crise convulsives. Il est peu probable que le propofol puisse déclencher des crises épileptiformes et les manifestations neurologiques constatées résultent en toute probabilité des mêmes mécanismes qui produisent de l'excitation à l'indication. Si les foyers épileptiques du cortex sont inhibés par une activité souscorticale, il serait alors possible pour le propofol d'inhiber la comitialité chez le malade susceptible. Il est toutefois inquiétant et unusuel de constater des mouvements athénoïdes et de l'opisthotomos après l'anesthésie. Ils ne surviennent à peu d'exceptions près qu'avec le propofol. En effet les phénothiazines peuvent produire des réactions dystoniques avec opisthotonos et mouvements athénoïdes comme on le voit avec la sédation au midazolam. Il est toutefois difficile de faire la relation de cause à effet entre le propofol et les réactions tardives décrites après l'usage du propofol. Ceci demeure sujet à spéculation.

\section{References}

1 Finley $G A$, MacManus B, Sampson SE, Fernandez $C V$, Retallick $R$. Delayed seizures following sedation with propofol. Can J Anaesth 1993; 40: 863-5.

2 Laycock GJA. Opisthotonos and propofol: a possible association (Letter). Anaesthesia 1988; 43: 257.

3 Wittenstein U, Lyle DJR. Fits after alfentanil and propofol (Letter). Anaesthesia 1989; 44: 532-3.

4 Paech MJ, Storey JM. Propofol and seizures (Letter). Anaesth Intensive Care 1990; 18: 585.

5 Saunders PRI, Harris MNE. Opisthotonus and other unusual neurological sequelae after outpatient anaesthesia. Anaesthesia 1990; 45: 552-7.

6 Shearer ES. Convulsions and propofol (Letter). Anaesthesia $1990 ; 45:$ 255-6.

7 Thomas $J S$, Boheimer NO. An isolated grand mal seizure 5 days after propofol anaesthesia (Letter). Anaesthesia 1991; 46: 508.

8 Harrison $J L$. Postoperative seizures after isoflurane anesthesia. Anesth Analg 1986; 65: 1235-6.

9 Waterhouse RG. Epileptiform convulsions in children following premedication with Pamergan SP100. $\mathrm{Br} \mathrm{J}$ Anaesth 1967; 39: 268-70.
10 Goroszeniuk T, Albin M, Jones RM. Generalized grand mal seizure after recovery from uncomplicated fentanyletomidate anesthesia. Anesth Analg 1986; 65: 979-81.

11 Kozody R, Ready LB, Barsa JE, Murphy TM. Dose requirement of local anaesthetic to produce grand mal seizure during stellate ganglion block. Can Anaesth Soc J 1982; 29: 489-91.

12 Pagden D, Halaburt AS, Wirpszo R, Karyn A. Posterior dislocation of the shoulder complicating regional anesthesia. Anesth Analg 1986; 65: 1063-5.

13 Stevens JE, Fujinaga $M$, Oshima $E$, Mori $K$. The biphasic pattern of the convulsive property of enflurane in cats. $\mathrm{Br} \mathrm{J}$ Anaesth 1984; 56: 395-403.

$14 \mathrm{Ng}$ ATH. Prolonged myoclonic contractions after enflurane anaesthesia-a case report. Can Anaesth Soc J 1980; 27: 502-3.

15 Grant IS. Delayed convulsions following enflurane anaesthesia. Anaesthesia 1986; 41: 1024-5.

16 Scott JC, Sarnquist FH. Seizure-like movements during a fentanyl infusion with absence of seizure activity in a simultaneous EEG recording. Anesthesiology 1985; 62: 812-4.

17 Milne B, Cervenko FW, Jhamandas KH. Physical dependence on nitrous oxide in mice: resemblance to alcohol but not to opiate withdrawal. Can Anaesth Soc J 1981; 28 : 46-50.

18 Manson HJ, Dyke G, Melling J, Gough M. The effect of naloxone and morphine on convulsions in mice following withdrawal from nitrous oxide. Can Anaesth Soc J 1983; 30: 28-31.

19 Glen JB, Hunter SC, Blackburn TC, Wood P. Interaction studies and other investigations of the pharmacology of propofol ("Diprivan”). Postgrad Med J 1985; 61 (Suppl 3): 7-14.

20 Lowson S, Gent JP, Goodchild CS. Anticonvulsant properties of propofol and thiopentone: comparison using two tests in laboratory mice. Br J Anaesth 1990; 64: 59-63.

21 Al-Hader $A F$, Hasan $M$, Hasan $Z$. The comparative effects of propofol, thiopental, and diazepam, administered intravenously, on pentylenetetrazol seizure threshold in the rabbit. Life Sci 1992; 51: 779-86.

22 Rampton AJ, Griffin RM, Stuart CS, Durcan JJ, Huddy $N C, A b b o t t M A$. Comparison of methohexital and propofol for electroconvulsive therapy: effects on hemodynamic responses and seizure duration. Anesthesiology 1989; 70: 412-7.

23 Mitchell P, Torda T, Hickie I, Burke C. Propofol as an anaesthetic agent for ECT: effect on outcome and length of course. Aust N Z J Psychiatry 1991; 25: 255-61.

24 Mackenzie SJ, Kapadia F, Grant IS. Propofol infusion for control of status epilepticus. Anaesthesia 1990; 45: 1043-5.

25 Peduto VA, Concas A, Santoro G, Biggio G, Gessa GL. Biochemical and electrophysiologic evidence that propofol 
enhances GABAergic transmission in the rat brain. Anesthesiology 1991; 75: 1000-9.

26 Dolin SJ, Smith MB, Soar J, Morris PJ. Does glycine antagonism underlie the excitatory effects of methohexitone and propofol? Br J Anaesth 1992; 68: 523-6.

27 Purdie JAM, Cullen PM. Brainstem auditory evoked response during propofol anaesthesia in children. Anaesthesia 1993; 48: 192-5.

28 Seifert HA, Blouin RT, Conard PF, Gross JB. Sedative doses of propofol increase beta activity of the processed electroencephalogram. Anesth Analg 1993; 76: 976-8.

29 Janti V, Yli-Hankala A, Baer GA, Porkkala T. Slow potentials of EEG burst suppression pattern during anaesthesia. Acta Anaesthesiol Scand 1993; 37: 121-3.

30 Borgeat A, Dessibourg C, Popovic V, Meier D, Blanchard $M$, Schwander $D$. Propofol and spontaneous movements: an EEG study. Anesthesiology 1991; 74: 24-7.

31 Borgeat A, Wilder-Smith OHG, Despland PA, Ravussin P. Spontaneous excitatory movements during recovery from propofol anaesthesia in an infant: EEG evaluation. $\mathrm{Br} \mathbf{J}$ Anaesth 1993; 70: 459-61. 